\title{
Angels, Insects, and Weather ${ }^{1}$
}

\author{
A. H. LaGrone, A. P. Deam, and G. B. Walker \\ Contribution From Electrical Engineering Research Laboratory, The University of Texas, Austin, Tex.
}

(Received April 8, 1964)

\begin{abstract}
This paper includes the results of a brief observation period with an M-33, X-band radar. Data presented are those obtained by pointing the radar vertically and observing the radar echoes as the targets drifted through the radar beam. Film recording of the data was used. Theoretical consideration of the radar problem for the various atmospheric phenomena and for animal-type sources was made to establish signal strength limits on the expected signal and is reported.
\end{abstract}

\section{Introduction}

It is well known that radar echoes can and have been received from optically invisible targets in the troposphere. Such targets are generally referred to as angels. Targets of this type have been observed and studied by researchers for the past 25 years or more. No model of the troposphere has ever been measured that could satisfactorily explain the origin of all such signals. Dr. V. G. Plank's report and summary published in 1956 [Plank, 1956], and Dr. David Atlas' report and summary in 1959 [Atlas, 1959], provide an excellent historical review of the angel-type work done in the past. Recent papers on the subject are to be found in the current technical literature.

This paper reports a number of angel-type measurements made at Austin, Tex., at X-band frequencies. Some angels were observed as they drifted through the narrow beam of a vertically looking radar while others were tracked to determine their course, elevation, and velocity. Radar cross sections were also measured.

The theoretical backscatter cross section for a variety of possible angel-type targets was computed in order to establish some kind of a limit on the signal amplitudes to be expected. Existing theories and models were used in conjunction with the characteristics of the radar antenna and receiver in the computations.

\section{Calculation of the Backscatter Cross Section}

The principal sources of radar echoes in the troposphere were deemed to be: (1) extended horizontal layers in the index of refraction structure of the troposphere, (2) turbulence in the index of refraction of the troposphere, (3) insects, (4) birds, and (5) certain clouds. Some of these sources were, of course, optically observable and others were not.

1 Paper presented at the 1963 fall URSI meeting, December 9-11, 1963, at the University of Washington, Seattle, Wash. Work sponsored by the National Science Foundation under Grant G 22115, and Air Force Cambridge Research Laboratories under Contract A F 19(604)-8038.

The X-band radar characteristics were as follows:

Peak power
Pulse length
Pulse rate
Receiver sensitivity

Antenna
$250 \mathrm{~kW}$

$0.25 \mu \mathrm{s}$

$1000 \mathrm{pps}$

$-98 \mathrm{dBm}$ for range lock. (This was increased, to $-110 \mathrm{dBm}$ by film integration techniques for visual purposes.)

Metal plate lens, $1.83 \mathrm{~m}$ in diameter, with a gain of $39.6 \mathrm{~dB}$ above an isotropic radiator. Beamwidth $=1.25^{\circ}$.

\subsection{For Layers}

Starting with the layer phenomena, assuming infinite horizontal extent of the layer, vertical incidence of the signal, and with Bauer's work [1956], an equation was obtained for the power returned to the radar receiver [Deam, Walker, and LaGrone, 1963]. This equation showed a direct dependence of the power returned on $\lambda^{4}$ and index of refraction gradient squared, i.e. $(\Delta n / t)^{2}$.

The importance of the index of refraction gradient was quite obvious. To compute an example believed to be representative of the local geographical area, i.e., the Southwest United States, and one that had been reliably measured [Deam and Walker, 1963] a value of one $N$-unit in $2 \mathrm{ft}$ was assumed. Using such a value and the constants for the M-33 radar, the power returned was found to be

$$
P_{R}=\frac{7.3 \times 10^{-8}}{R^{2}} \text { milliwatts, }
$$

where

$$
R=\text { range in meters. }
$$

If a range of $1 \mathrm{~km}$ is assumed, the received power is $7.3 \times 10^{-14} \mathrm{~mW}$, which is approximately $20 \mathrm{~dB}$ below the estimated sensitivity of the M-33 radar receiver using film integration techniques. As stated in the radar characteristics, film integration increased the system visual sensitivity by about $12 \mathrm{~dB}$ or to $-110 \mathrm{dBm}$. 
In order to detect a 2 -ft layer at a height of $1 \mathrm{~km}$, a refractive index change of nearly $10 \mathrm{~N}$-units would be required, or a one $N$-unit change would need to occur in $7 \mathrm{~cm}$. If the transitory layer filled approximately one Fresnel zone in the horizontal plane, the reflected radar energy would be increased by a factor of approximately four [Atlas, 1960], which is still well below the capability of the present receiving equipment.

Since $\lambda^{4}$ dependence was indicated in the equation for the power reflected from a layer, it was interesting: to determine the wavelength, other factors remaining constant, at which the assumed layer would just be visible. It turned out to be approximately $10 \mathrm{~cm}$.

\subsection{For Turbulence}

In estimating signal strengths to be expected as a result of turbulence in the troposphere, power density spectra previously measured and reported at the University of Texas can be used [Walker, 1960]. These must be extrapolated to the preferred wave number, however, which raises a question as to the validity of the subsequent solution. Using the work of Megaw [1957], the power density spectra and the $\mathrm{X}$-band radar characteristics, a curve of power return due to turbulence in the troposphere is computed and shown in figure 1.

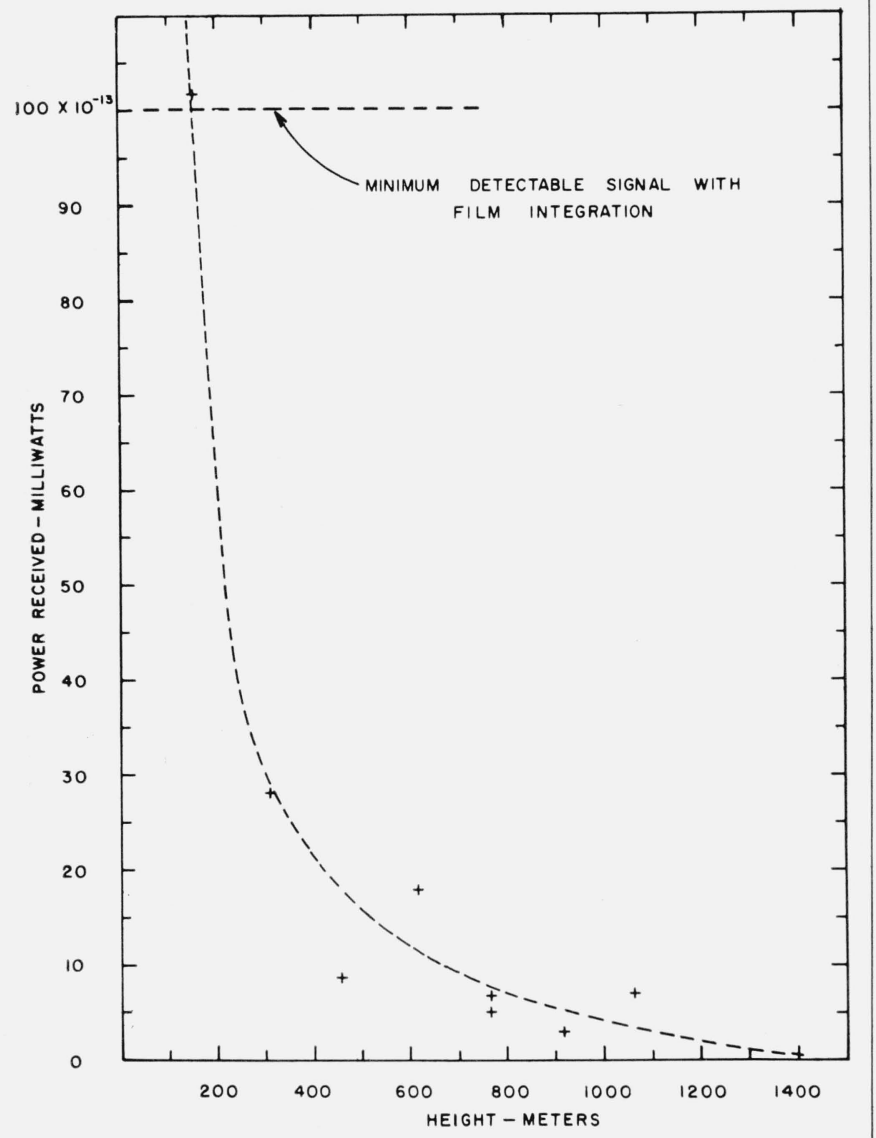

Figure 1. Power returned due to turbulence in the troposphere.
It can be seen that the predicted signal is well below the receiver capability at all but the lowest target heights. It is believed that this curve presents an optimistic view of the possible power returned.

\subsection{For Insects}

In considering insects as possible sources of angeltype echoes, it is necessary to estimate their backscattering cross section. In this connection Tolbert, Straiton, and Britt [1958] reported that a common housefly is the approximate equivalent of a $3 \mathrm{~mm}$ drop of water. Stephens [1961], calculates the backscattering cross section for such a drop of water, at $3.2 \mathrm{~cm}$ wavelength, to be $0.002 \mathrm{~cm}^{2}$. For such a target, the range producing a signal equal to the noise in the $\mathrm{M}-33$ radar, using film integration, is $1390 \mathrm{~m}$. Many flying insects, such as butterflies, locusts, etc., have larger backscattering cross sections and would thus be detectable at greater ranges.

\subsection{For Birds}

Equivalent cross sections for birds may well exceed the cross section of a common housefly by a factor of $10^{4}$. This being so, detectable returns from birds could well be expected at ranges up to $13,900 \mathrm{~m}$. It is evident from these calculations that, both with and without film integration, there is a high probability of detectable returns from flying animal matter. Such targets could be tracked with the M-33 $\mathrm{X}$-band radar.

\subsection{For Clouds}

Cloud droplets would be a possible source of incoherent angel-type signals. Using the radar cross section of a waterdrop [Stephens, 1961] and the drop size distribution for fair weather cumulus and cumulus-congestus clouds [Mason, 1957], cross sections were computed. These values yield minimum detectable signals (without film integration) for the M-33 $\mathrm{X}$-band radar at ranges of approximately $15 \mathrm{~km}$ for the cumulus congestus and about $5 \mathrm{~km}$ for the fair weather cumulus clouds. A rainfall rate of $1 \mathrm{~mm} / \mathrm{hr}$ gives a power return that will be detectable at ranges up to about $80 \mathrm{~km}$.

With film integration techniques, the fair weather cumulus gives a detectable return at a range of 16 $\mathrm{km}$ and the cumulus congestus gives a detectable return at ranges up to $40 \mathrm{~km}$. It is presupposed that the scattering volumes fill the main beam of the radar antenna in these examples.

\section{Experimental Measurements}

In this experiment, the first actual radar observations were made in the vicinity of the Electrical Engineering Research Laboratory building at Austin, Tex., with the antenna pointed vertically and not lobe scanning. It was immediately apparent that signals from surface targets returned via the side lobes would constitute a major problem in the detection of the small signals expected. Figure 2 is a photograph 


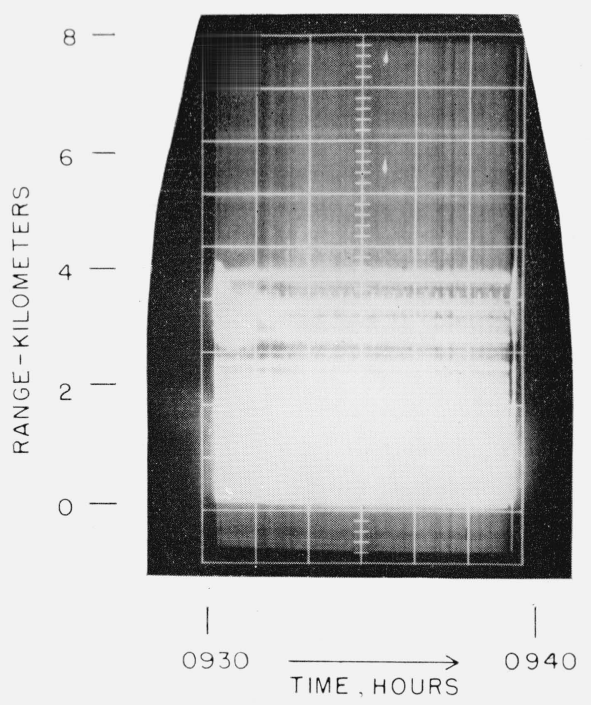

Figure 2. Surface targets detected through side lobes of vertically pointed radar antenna.

taken to illustrate this point. Although this picture was made at a later date, for record, it shows the original side lobe problem.

The photograph shows clearly that strong side lobe returns occur out to ranges of the order of $3700 \mathrm{~m}$ and that ground clutter saturates the receiver out to $1800 \mathrm{~m}$. Figure 2 also shows a passing cloud at about $3000 \mathrm{~m}$ which was clearly visible to the unaided eye.

The photograph shown in figure 2 and all subsequent angle photographs were made with a $535 \mathrm{~A}$ oscilloscope. The video was fed to the intensity grid of the cathode ray tube in the oscilloscope. The horizontal sweep was synchronized to the radar and a motor-potentiometer-battery arrangement on the vertical amplifier moved the trace across the film. The pictures as presented here are rotated counterclockwise through $90^{\circ}$ relative to the oscilloscope face. Each 10-min picture is composed of about 600,000 individual traces.

It was obvious from the early measurements that a better observation site had to be found. Dotlike echoes were observed in the very first measurements but due to the side lobe problem there was considerable question as to whether they were originating from small targets in the main beam or from larger targets in the side lobes.

A new location was found that was considerably better than the laboratory site. It was an abandoned gravel pit that was deep and narrow. The lens antenna was approximately at local ground level when the radar van was positioned in the pit.

The first pictures taken at the new site showed that ground-clutter saturation had been reduced to approximately $600 \mathrm{~m}$ and that no fixed ground targets appeared outside of the ground clutter.
The new site was approximately $200 \mathrm{~m}$ from a heavily traveled highway but was very well concealed by a forest of cedar and live oak trees. Moving automobiles on the highway were never detected. Had they been, they would have appeared as paraboliclike tracks with the parabola opening upwards, and no such tracks were ever observed.

All tracking measurements of dot angels were accompanied by a balloon flight in order that wind velocities could be estimated. The time differential between the balloon flight and the angel measurements was generally less than $1 \mathrm{hr}$. On two occasions, $15.25 \mathrm{~cm}$ aluminum spheres were flown for calibration purposes. All dot angel cross sections were evaluated using results from the aluminum sphere flights. A 20-power theodolite was attached to the radar antenna and boresighted to give better optical tracking capability than that available with the M-33 periscope.

Some large birds were tracked to establish the time characteristics of signals from such targets. Such a signal record is shown in figure 3 . The equivalent cross section of the bird in this recording is about the same as that quoted elsewhere for sea gulls, i.e., about 25 to $250 \mathrm{~cm}^{2}$ The bird in this case is a turkey buzzard.

\section{Discussion of Results}

Figure 4 shows a series of photographs taken through the sunrise period on 9 August 1963. The photographs were all taken at the gravel pit site. In the 6-hr period prior to the taking of this series of photographs there was light but general thundershower activity in the region. There were two cloud layers, 0.7 altocumulus at $3.7 \mathrm{~km}$ and 0.3 cumulus at $1.5 \mathrm{~km}$. The upper clouds are clearly visible in some of the photographs. The lower clouds had all but disappeared at the time the photographs were taken.

The haze layer extending from approximately 0.9 to $1.2 \mathrm{~km}$ was something of a mystery. It does not appear to be the result of the recording technique; on the other hand, its range does not change in figure $4 \mathrm{~d}$ as the antenna is tilted in tracking a dot angel. This behavior makes one strongly suspect that maybe it is a recording error.

The linelike returns in figure $4 \mathrm{e}$ are believed to be semiextended layers. Automobile's reflections, as stated earlier, can well be discounted because of the nature of the tracks and the fact that the lines were not always present during times of high traffic density.

The increase in dot angel density with the coming of sunrise is spectacularly shown. The dot type of return was generally visible at all hours of the day and night but the density was always greater in the daytime. It seems quite likely that some of these returns could be large insects or birds although their true origin is unknown at the moment. The signal strengths generally peaked at about -80 to $-90 \mathrm{dBm}$. At the gravel pit site, almost without exception, there was a sharp decrease in the density 


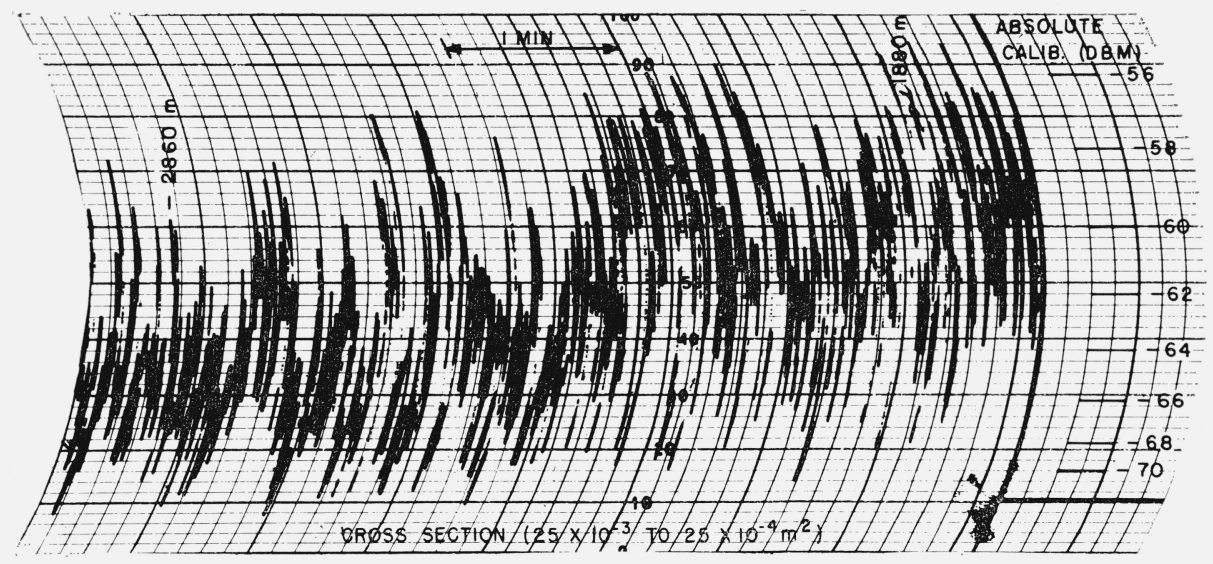

FiguRE 3. Time characteristics of the signal from a large bird (turkey buzzard).
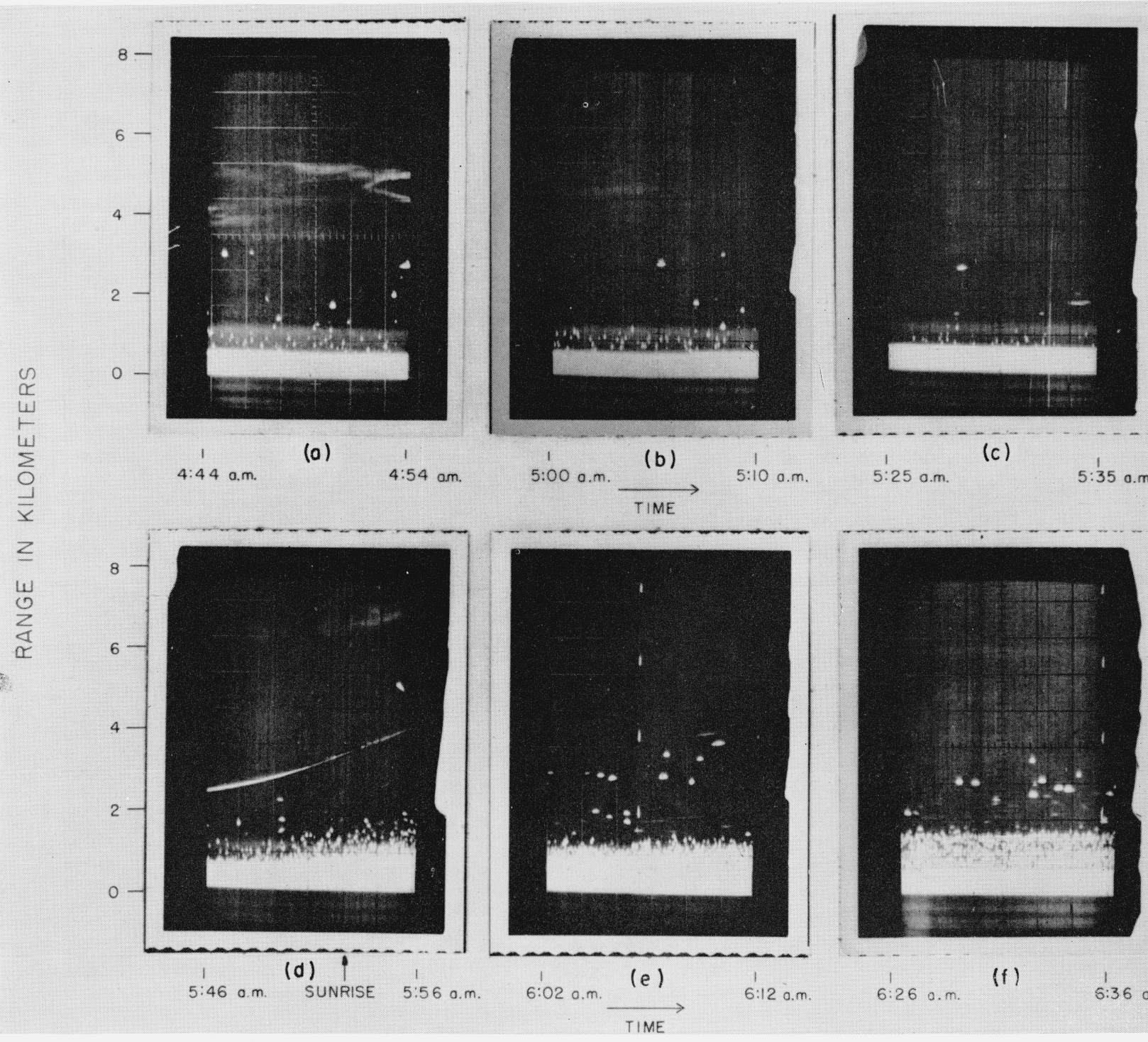

(c)

5:35 a.m.

5:25 a.m

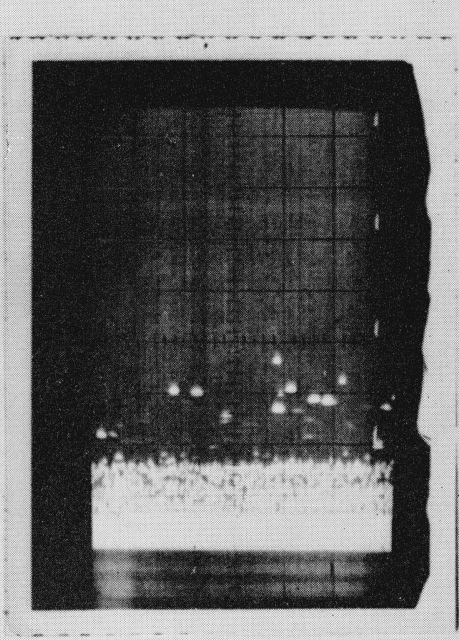

(f)

6:36 a.m

FIGURE 4. Variability in the number of "angel" echoes with time around sunrise. 

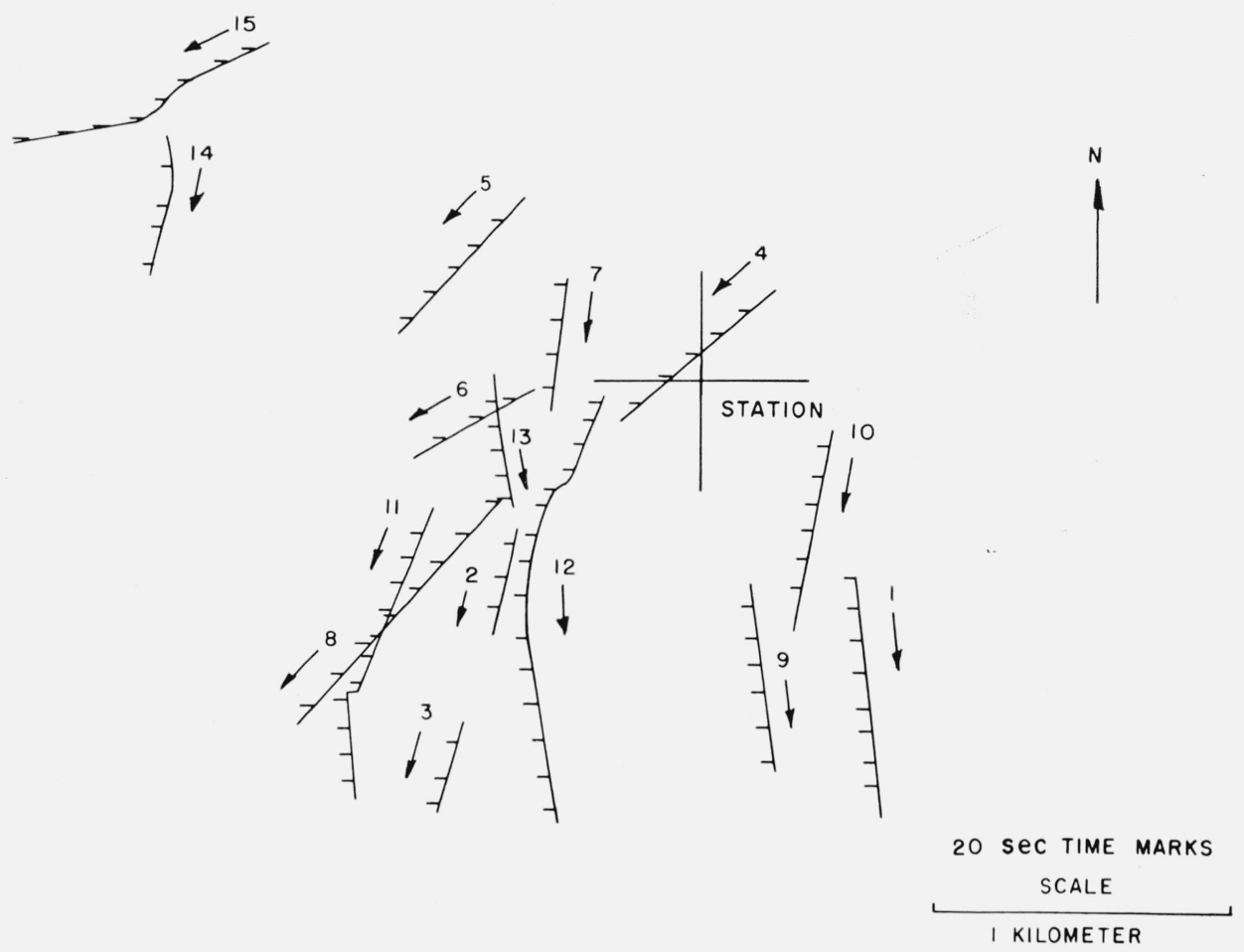

Figure 5. Horizontal track characteristics (9 Sept.1963-afternoon).

of the dot angels with altitude. This is clearly shown in figure $4 \mathrm{f}$ at approximately $1.5 \mathrm{~km}$. The dot angel echoes as viewed on the "A" scope and in the range gate did not differ materially in shape from the transmitted pulse. This type echo was observed as high as $5.8 \mathrm{~km}$.

Many of the dot angel-type targets were tracked for record. Similar tracks have been reported by other researchers [Roelofs, 1963], and the character of the tracks reported here do not differ grossly from theirs. Tracks are presented in the form of projections onto the horizontal plane, as height versus range tracks, and as plots of horizontal velocity vectors along with wind vectors.

Dot angel tracks are presented in figures 5,6 and 7 for the afternoon of 9 September 1963. The angels are numbered the same on each figure for identification, i.e., No. 1 , figure 5 is the same as No. 1, figure 6 and as No. 1, figure 7 . It should be noted in figure 6 that some angels, No. 4, for example, are tracked "in" to a minimum range and "out", as the range increases. This shows very clearly in the horizontal track of No. 4, figure 5.

Characteristics of the dot angels tracked in figures 5, 6 and 7 and the many other dot angels tracked but not reported here, can be summarized as follows:

1. None of the dot angel tracks were associated with visual observation of a target. (If the target was visually identified, as, for example, the turkey buzzard, the target was not designated a dot angel.)

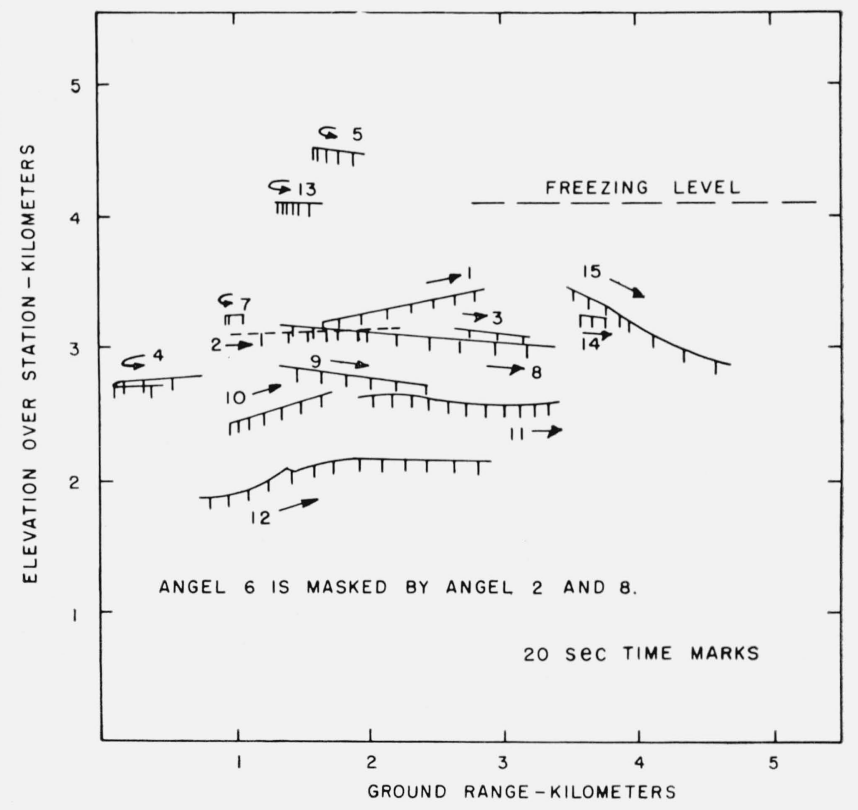

Figure 6. Vertical motion (9 Sept. 1963-afternoon).

2. Over an observation period of approximately 60 days, dot angels were found on all days but in varying abundance. Dot angels were observed at night as well as during the day.

3. Tracks indicate that nearly all dot angels had a velocity different from the wind. 


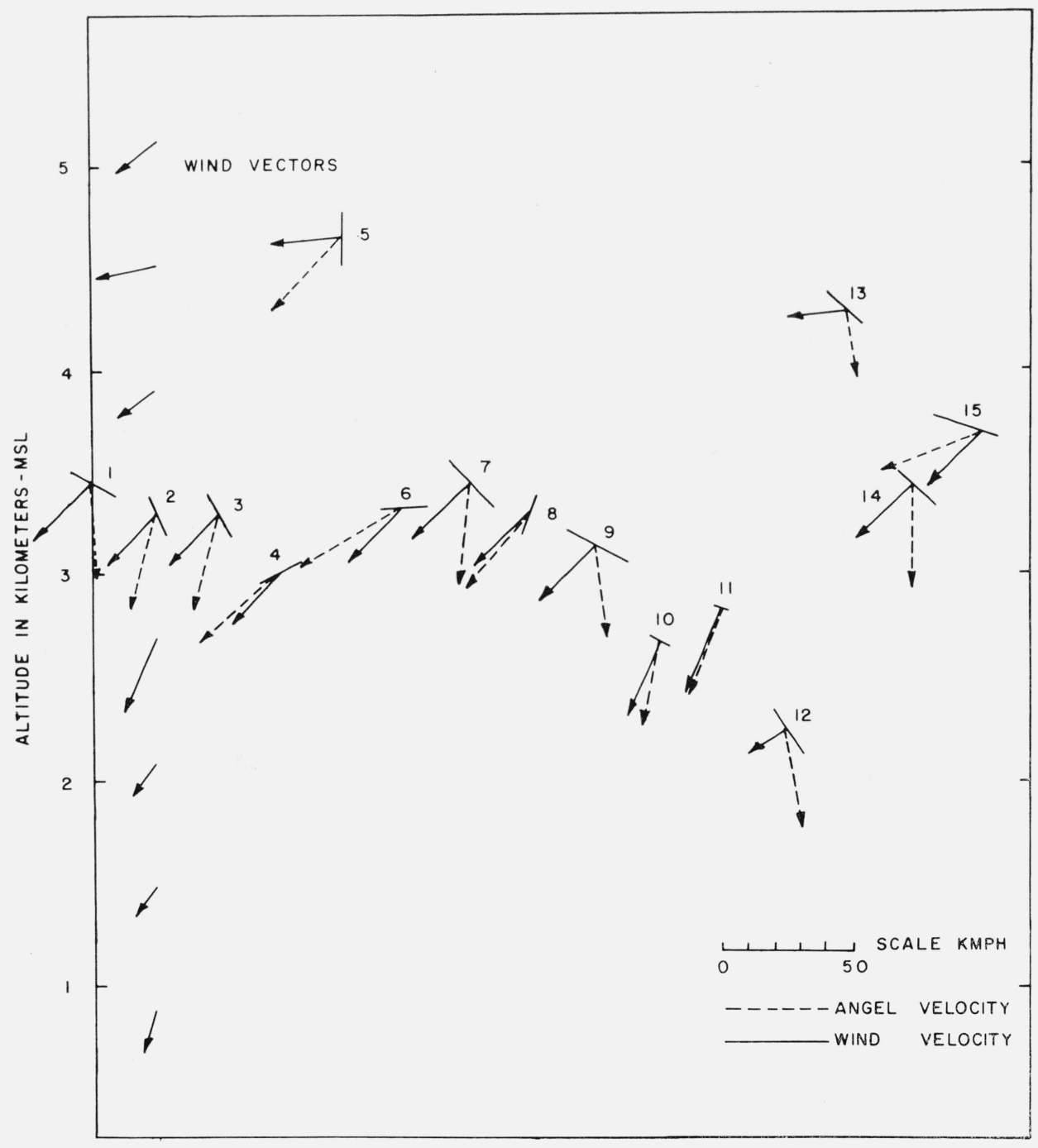

Figure 7. Angel velocity characteristics (9 Sept. 1963-afternoon).

4. Vertical velocities were observed and measured; there was some indication that they were greater on hotter days.

5. Median cross section for all dot angels was of the order of $1 \mathrm{~cm}^{2}$, with values ranging from 0.1 to $10 \mathrm{~cm}^{2}$.

6. Many of the tracked dot angel echoes did not show the signal strength variation with time observed for large birds.

7. All dot angel echoes had essentially the same shape as the transmitted pulse.

8. Dot angel cross sections appeared to be aspect dependent. A feature common to many dot angels was a minimum equivalent cross section when the electric vector became normal to the track. The radar's magnetic vector was always in the horizontal plane at the ranges encountered.

9. Signal strength dependence on range to the minus fourth power (i.e., $R^{-4}$ ) was found to be the exception rather than the rule. The signal strength dropped at a rate greater than $R^{-4}$ indicated that it should. This would tend to strengthen the aspect dependent characteristics noted above.

10. Dot angels echoes were seldom observed above the freezing level. (Freezing level was determined from weather bureau data.)

11. Dot angels were found not to be sensitive to small changes in frequency.

12. The radar, while tracking one angel, would often reject it in favor of another angel coming into the range gate, particularly if the second angel had a larger cross section. Horizontal tracks changed abruptly in many instances with little change in altitude.

13. Straight line tracks in the horizontal plane were found in the majority of cases. Available track time was generally less than $10 \mathrm{~min}$ although some dot angels were tracked longer. 


\section{Conclusion}

(a) Under proper operating conditions, the M-33 $\mathrm{X}$-band radar can be used advantageously as an atmospheric probe.

(b) Preliminary investigation indicates that it is highly desirable to make simultaneous S-band measurements to establish wavelength dependence for echoes arising from various targets.

(c) It has been shown that there exists a high probability that echoes arising from insects and birds are to be detected at times. It is assumed that such echoes would have much the same characteristics as those exhibited by the dotlike returns reported herein.

(d) It appears, on occasion, that returns have been observed from extended layers of large index of refraction gradient or perhaps cloud-air interfaces (platelike targets).

(e) No returns were ever observed that could positively be attributed to normal turbulence in the troposphere.

(f) The aspect-dependent characteristic observed in some echoes should be investigated further. It is suggested that simultaneous observations of this type of return with orthogonal polarization and two wavelengths may be of value.

\section{References}

Atlas, D. (1959), Radar studies of meteorological angel echoes, J. Atmospheric Terrest. Phys. 15, 262-287.

Atlas, D. (1960), Possible key to the dilemma of meteorological angel echoes, J. Meteorol. 17, No. 2, 95-103.

Bauer, J. R. (Sept. 24, 1956), The suggested role of stratified elevated layers in transhorizon short-wave radio propagation, Tech. Rept. No. 124, MIT, Lincoln Laboratory, Cambridge, Mass.

Deam, A. P., and G. B. Walker (Feb. 28, 1963), High altitude microvariations within the atmosphere's radio refractiveindex profile, EERL Rept. No. 6-52, p. 18, The University of Texas, Austin, Tex.

Deam, A. P., G. B. Walker, and A. H. LaGrone (Nov. 30, 1963), Observations at and near vertical incidence with an M-33 radar, EERL Rept. No. 6-56, The University of Texas, Austin, Tex.

Mason, B. J. (1957), The Physics of Clouds, p. 93 (Oxford, Clarendon Press).

Megaw, E. C. S. (1957), Fundamental radio scatter propagation theory, Proc. IEE 104, Part C, 441.

Plank, V. G. (1956), A meteorological study of radar angels, Geophysical Research Paper No. 52, Bedford, Mass., Air Force Cambridge Research Center.

Roelofs, T. H. (1963), Characteritics of trackable radar angels, Research Report RS 137, Ithaca, New York, Cornell University, Center for Radio Physics and Space Research.

Stephens, J. J. (June 1961), Radar cross sections for water and ice spheres, J. Meteorol. 18, No. 3, 348-359.

Tolbert, C. W., A.W. Straiton, and C. O. Britt (Feb. 21, 1958), Phantom radar targets at millimeter radio wavelengths, EERL Rept. No. 99, The University of Texas, Austin, Tex.

Walker, G. B. (Sept. 15, 1960), Single and dual cavity measurements of ground and airborne index of refraction measurements at 400 meps, EERL Rept. No. 6-38, The University of Texas, Austin, Tex. 